\title{
Mean field analysis for inhomogeneous bike sharing systems
}

\author{
Christine Fricker ${ }^{1 \dagger}$ and Nicolas Gast ${ }^{2 \ddagger}$ and Hanene Mohamed ${ }^{3 \S}$ \\ ${ }^{1}$ INRIA Paris-Rocquencourt, Domaine de Voluceau, 78153 Le Chesnay, France \\ ${ }^{2}$ EPFL, LCA2, CH-1015 Lausanne \\ ${ }^{3}$ Université Paris Ouest, Bat G, 200 av. de la République, 92001 Nanterre CX, France \\ received 2012-02-01, revised $5^{\text {th }}$ April 2012, accepted tomorrow.
}

In the paper, bike sharing systems with stations having a finite capacity are studied as stochastic networks. The inhomogeneity is modeled by clusters. We use a mean field limit to compute the limiting stationary distribution of the number of bikes at the stations. This method is an alternative to analytical methods. It can be used even if a closed form expression for the stationary distribution is out of reach as illustrated on a variant. Both models are compared. A practical conclusion is that avoiding empty or full stations does not improve overall performance.

\section{Introduction}

Motivation. Bike sharing systems have generated research interest recently as such programs have been launched in many cities around the world. The concept is quite simple: the user takes a bike to a station, uses it and returns it at a station of his choice.

Despite their success, it is commonly observed that they do not behave very well. Users have to address the lack of resources, finding an empty station when in need of a bike, or a full station when returning a bike. The intuition is that the system is highly inhomogeneous, with very different arrival rates and destinations, between housing and working areas for instance, or up-hill and down-hill stations. These parameters may also vary with the time of day (see Borgnat et al.[1]), but this issue is not considered here.

In the literature on this topic, few analytical models have been proposed, especially stochastic models, mainly because this leads to complex model that are are difficult to analyze. Nevertheless, such mathematical models could give insight on the behavior of the system or study different incentives algorithms for taking or returning bikes, designed to improve overall performance.

Goal. The paper deals with a stochastic model taking into account the finite number of bike posts at the stations. To our knowledge, it is the first attempt to analyze the problem of returning the bikes. Our aim is to study the impact of system inhomogeneity. We use an asymptotic approach, studying a system in which the number of stations and the fleet get large together. The proportion of bikes per station and the

\footnotetext{
†Email: christine.fricker@inria.fr.

¥Email: nicolas.gast@epfl. ch.

$\S$ Email: hanene.mohamed@gmail.com
}

subm. to DMTCS (C) by the authors Discrete Mathematics and Theoretical Computer Science (DMTCS), Nancy, France 
number of spots per station, which are two key parameters of the system, remain fixed. This asymptotic study is quite adequate for bike sharing systems, that have hundreds or thousands of stations.

Main result. The main result is the limiting stationary distribution of the number of bikes in the stations as the system gets large. The proof is based on mean field analysis. It amounts to considering the empirical measure process. In the special case of homogeneous model, it is simply the process $Y(t)=$ $\left(Y_{0}(t) \ldots Y_{K}(t)\right)$ where $Y_{i}(t)$ is the proportion of stations with $i$ bikes at time $t$. It converges when the system gets large to a deterministic dynamical system, solution of an ODE. Using a Lyapunov function for the dynamical system, we show that it has a unique attractor. This yields the convergence of the stationary distribution to this fixed point. Notice that it is often difficult to exhibit such a function.

The challenge is to deal with inhomogeneity of the system that makes the analysis of the system of differential equations difficult. While other works use numerical simulations of the system [6], we obtain analytically the limiting stationary distribution, by characterizing the equilibrium point and providing a Lyapunov function. Despite the cluster structure, our method uses a compact way to write the differential equation.

Mean field limit is an indirect way to obtain the limiting stationary distribution: from a system of fixed size, we take the limit as the size gets large, then the limit as time tends to infinity, identify the limits, and of course prove convergences. In our model, say I, the stationary distribution for a fixed size has a closed form expression. A more direct approach would be to obtain an asymptotic from this expression by an analytical method. The benefit of mean field analysis is that it works for models where the stationary probability measure is out of reach. As an example, we study an model where users take bikes from nonempty stations and return them directly to non-saturated stations. For this new model, say model II, the same results are only partially proved. However its homogeneous version is completely understood.

Impact on performance. These results allow us to study the performance of large bike sharing systems. The limiting probability that a station is empty or full is taken as the performance measure. In the homogeneous case, the optimal proportion of bikes per station is slightly more than half the capacity of a station. Moreover performance is very close for both models I and II for a large range of values around this optimum. When the system is inhomogeneous, there is a proportion of bikes per station $s$, depending on each cluster, which minimizes the ratio of problematic stations inside a cluster. However, even if, for a given proportion $s$, performance is not bad in one cluster, the ratio of problematic stations in the other clusters can be very high, and overall performance is then very poor. The main conclusion of the study is that performance collapses due to heterogeneity.

Related work. As noted above, studies of such systems are rare. Bike sharing systems can be modelled as closed stochastic networks since the fleet is constant. If the capacity of stations is infinite, they are simply closed Jackson networks where the nodes are a mixture of one-server queues for the stations and infinite-server queues for the routes. The work by Malyshev and Yakovlev [9] on closed Jackson networks with one-server queues can be extended in this case. The limiting behavior of the network, namely condensation in some stations while the others exhibit a queue length with geometric distribution, can be similarly obtained. The technical arguments developed in [9] are analytical: from the product form of the stationary invariant measure for fixed size, a saddle point method is used (see [4] for an introduction) to obtain the asymptotic of the partition function. Note that George and Xia [7], in the context of bikesharing systems, provide a simpler proof in a more elementary framework: a case where the number of stations is fixed while the total fleet becomes large.

Mean field limits are applied to study a large class of models in statistical physics or applied probability. It has been also introduced in analysis of algorithms (see e.g. [10,11]). An issue raised in [12] (see also 
[3]) is to give an interpretation of Lyapunov functions related to relative entropy in some communication networks. It is pointed out in [12] that the special feature of the model is a strong requirement for obtaining the Lyapunov function. Our model has a similar behavior and provides new insights on that topic.

Outline of the paper. Section 2 gives model I description and the main result. Section 3 is devoted to its proof, based on a mean field approach. Model II is described and investigated in Section 4 and practical results about performance of both models are presented in Section 5.

\section{Model I: description and main result}

This section gives a description of model I and the main result. This model has reversible Markovian dynamics which allows to obtain the invariant distribution.

We consider a bike sharing system with $N$ stations and a fleet of $M$ bikes. Each station $i$ has a capacity $K_{i, N}$. The dynamics of the system is as follows. Users arrive at the stations according to independent Poisson processes with rate $\lambda_{i, N}$ at station $i$. When a user arrives at a station, if there is no available bike, then he leaves the system. Otherwise, he takes a bike and chooses station $j$ with probability $p_{j, N}$. The travel time has an exponential distribution with parameter $\mu_{N}$, whatever the station he comes from. When he arrives at station $j$, if there are less than $K_{j, N}$ bikes in this station, he returns his bike and leaves the system. If there are $K_{j, N}$ bikes (i.e. the station is full), the user chooses again a station say $k$ with probability $p_{k, N}$ and goes to this station. As before, it takes a time with exponential distribution with parameter $\mu_{N}$. He rides like this again until he can return his bike.

This model is a generalization of the homogeneous case where the arrival rates do not depend on the stations and where the user returns the bike at a station chosen at random. Even if the different probabilities to go from a station to another do not appear exactly, as a routing matrix, the popularity of a station is taken into account.

\subsection{Markovian reversible dynamics}

Let $N$ be fixed and $X_{i, N}(t)$ be the number of bikes at station $i, 1 \leq i \leq N$. The process $\left(X_{N}(t)\right)=$ $\left(X_{i, N}(t)\right)_{1 \leq i \leq N}$ is an irreducible Markov jump process on the finite state space

$$
\mathcal{S}_{N}=\left\{x=\left(x_{1}, \ldots, x_{N}\right) \in \mathbb{N}^{N}, 1 \leq x_{i} \leq K_{i, N}, \sum_{i=1}^{N} x_{i} \leq M\right\}
$$

thus it has a unique invariant probability measure $\pi_{N}$. The process is reversible, so it is easy to obtain $\pi_{N}$. Let $R_{i, N}=\mu_{N} p_{i, N} / \lambda_{i, N}$ be the utilization at station $i$. For $x$ in $\mathcal{S}_{N}$ and $|x|=\sum_{i=1}^{N} x_{i}$,

$$
\pi_{N}(x)=\frac{1}{Z_{N}} \frac{\prod_{i=1}^{N} R_{i, N}^{x_{i}}}{(M-|x|) !}
$$

where the normalizing constant is $Z_{N}=\sum_{x \in \mathcal{S}_{N}}\left(\prod_{i=1}^{N} R_{i, N}^{x_{i}}\right) /(M-|x|) !$.

\subsection{Main result}

It gives an asymptotic of the invariant measure $\pi_{N}$ for $N$ and $M$ large, with $M / N$ tending to $s>0$. Let $r_{i, N}=R_{i, N} / \max _{i} R_{i, N}$ be the relative utilization at station $i$. The following assumption is crucial: 
Assumption $W$. There exists $I$ a probability measure on $] 0,1] \times \mathbb{N}$ and $\Lambda>0$ such that, as $N$ tends to infinity,

$$
\frac{1}{N} \sum_{i=1}^{N} \delta_{r_{i, N}, K_{i, N}} \stackrel{(w)}{\longrightarrow} I \text { and } N R_{\max , N} \rightarrow \Lambda^{-1}
$$

where $w$ means the weak convergence of measures and $R_{\max , N}=\max _{i} R_{i, N}$.

Note that this assumption is fundamental for the convergence to the solution of the differential equations and is related to the system topology (see for illustration the examples above).

Theorem 1 Under assumption $(W)$, if I has a finite support, as $N$ and $M$ tend to infinity with $M / N$ tending to some s, the number of bikes at a station with parameters $r$ and $K$ has a geometric distribution $\nu_{\rho r, K}$ on $\{0, \ldots, K\}$ with parameter $\rho r$ where $\rho$ is the unique solution of

$$
s=\rho \Lambda+\int_{] 0,1] \times \mathbb{N}} m\left(\nu_{\rho r, K}\right) d I(r, K)
$$

where the mean of a random variable with distribution $\mu$ is denoted by $m(\mu)$.

Theorem 1 is proved in Section 3. Let us first give two examples.

\subsection{Examples}

To simplify, $\lambda_{i, N}, K_{i, N}, \mu_{N}$ and $N p_{i, N}$ do not depend on $N$. It can be straightforwardly generalized.

The homogeneous case. For each $i, K_{i}=K, \lambda_{i}=\lambda$ and $p_{i, N}=1 / N$ thus $r_{i, N}=1$. Assumption $(W)$ is obvious with $I=\delta_{1, K}$ and $\Lambda=\lambda / \mu$. Equation (2) rewrites

$$
s=\frac{\lambda}{\mu} \rho+m\left(\nu_{\rho, K}\right) .
$$

The cluster case. Assume that there are $C$ clusters and each cluster $c$ has $N_{c}$ stations with $N_{c} / N$ which tends to $\alpha_{c}$. Each station in cluster $c$ has the same parameters $\lambda_{c}, K_{c}$ and $p_{c, N}=\beta_{c} / N$. Thus each station in cluster $c$ has the same utilization $R_{c, N}$ with $N R_{c, N}=\mu \beta_{c} / \lambda_{c}$. Assumption $(W)$ holds with $I=\sum_{c=1}^{C} \alpha_{c} \delta_{r_{c}, K_{c}}$, with $r_{c}=\Lambda \mu \beta_{c} / \lambda_{c}$ where $\Lambda=1 / \max _{c}\left(\mu \beta_{c} / \lambda_{c}\right)$.

Equation (2) can be rewritten

$$
s=\rho \Lambda+\sum_{c=1}^{C} \alpha_{c} m\left(\nu_{\rho r_{c}, K_{c}}\right)
$$

which generalizes equation (3) obtained for the homogeneous case.

\section{A mean field analysis}

Subsection 3.1 is devoted to the proof of Theorem 1, divided into several steps. Some connections to an analytical method are given in 3.2 . 


\subsection{Mean field limit}

A simplification. For fixed $N$, the invariant measure $\pi_{N}$ given by equation (1) depends on the different parameters only by $R_{i, N}$. Thus, to obtain the limiting invariant measure, one can assume without loss of generality that $\mu_{N}=1$ and a uniform routing $p_{i, N}=1 / N$. In what follows, we assume that $K_{i, N}=K_{N}$ to avoid cumbersome notations but it remains valid replacing $r$ by $(r, K)$ and proves Theorem 1 .

The empirical measure process. Due to heterogeneity with respect to the $N$ stations, the quantity of interest is the following empirical distribution of the stations as function of time

$$
Y^{N}(t)=\frac{1}{N} \sum_{i=1}^{N} \delta_{\left(r_{i, N}, X_{i, N}(t)\right)}
$$

The empirical measure process $\left(Y^{N}(t)\right)$ is a Markov process on the finite state space

$$
\mathcal{Y}^{N}=\left\{y=\frac{1}{N} \sum_{i=1}^{N} \delta_{\left(r_{i, N}, n_{i}\right)},\left(n_{i}\right)_{1 \leq i \leq N} \in \mathbb{N}, n_{i} \leq K_{i, N}, \sum_{i=1}^{N} n_{i} \leq M\right\},
$$

included in $\mathcal{Y}=\mathcal{P}([0,1] \times \mathbb{N})$, the set of probability measures on $] 0,1] \times \mathbb{N}$, with transitions

$$
\begin{array}{lr}
y \rightarrow y+\frac{1}{N}\left(\delta_{(r, n-1)}-\delta_{(r, n)}\right) & \frac{1}{r R_{\max , N}} y(r, n) 1_{n>0} \\
y \rightarrow y+\frac{1}{N}\left(\delta_{(r, n+1)}-\delta_{(r, n)}\right) & N y(r, n)\left(M-\sum_{\left(r^{\prime}, n^{\prime}\right)} n^{\prime} y\left(r^{\prime}, n^{\prime}\right)\right) 1_{n<K}
\end{array}
$$

The first transition is an arrival at a station with utilisation $r$ and with $n$ bikes, $n>0$. The second one is a return of a bike in a station with utilisation $r$ and with $n$ bikes, $n<K$.

Remark. The first marginal of $Y^{N}(t)$ is $Y_{1}^{N}(t)=\frac{1}{N} \sum_{i=1}^{N} \delta_{r_{i, N}}$ which is deterministic. Thus, $(W)$ is necessary for the convergence of process $Y^{N}$.

Convergence to the dynamical system. Under assumption $(W)$, as $I$ has a finite support, by the same arguments as for an homogeneous model (see for example Darling and Norris [2]), $Y^{N}$ converges in distribution to a dynamical system, unique solution of the following differential equation

$$
\dot{y}=\iint_{[0,1] \times\{0, \ldots, K\}}\left(\frac{\Lambda}{r}\left(\delta_{(r, n-1)}-\delta_{(r, n)}\right) 1_{n>0}+1_{n<K}\left(\delta_{(r, n+1)}-\delta_{(r, n)}\right)\left(s-m\left(y_{2}\right)\right)\right) d y(r, n)
$$

where $m\left(y_{2}\right)=\iint n d y(r, n)$.

Equilibrium point for the dynamical system. If $\bar{y}$ in $\mathcal{Y}$ is an equilibrium point for the dynamical system, applying the right-hand side of equation (5) to $A \times\{p\}, A \subset] 0,1], p \in\{0, \ldots, K\}$,

$$
\begin{aligned}
0 & =\left(1_{p>0} \bar{y}(A \times\{p-1\})-1_{p<K} \bar{y}(A \times\{p\})\right)\left(s-m\left(\bar{y}_{2}\right)\right) \\
& +1_{p<K} \iint_{A \times\{p+1\}} \frac{\Lambda}{r} d \bar{y}(r, n)-1_{p>0} \iint_{A \times\{p\}} \frac{\Lambda}{r} d \bar{y}(r, n) .
\end{aligned}
$$


It means that, for $r>0, \bar{y}(r,$.$) is the invariant measure of a M / M / 1 / K$ queue with arrival to service rate ratio $r\left(s-m\left(\bar{y}_{2}\right)\right) \Lambda^{-1}$. Thus, given $r, \bar{y}(r,$.$) is a geometrical distribution on \{0, \ldots, K\}$ with parameter $r \rho(\bar{y})$ where $\rho(y)$ is defined on $\mathcal{Y}$ by

$$
\rho(y)=\left(s-m\left(y_{2}\right)\right) \Lambda^{-1} .
$$

Recall that the geometric distribution on $\{0, \ldots, K\}$ with parameter $a$ is denoted by $\nu_{a, K}$. One gets that, for the equilibrium point $\bar{y}$,

$$
m\left(\bar{y}_{2}\right)=\int m\left(\nu_{r \rho(\bar{y}), K}\right) d I(r)
$$

which, plugging it in equation (6), gives

$$
\rho(\bar{y})=\left(s-\int m\left(\nu_{r \rho(\bar{y}), K}\right) d I(r)\right) \Lambda^{-1} .
$$

Thus, to prove the existence and uniqueness of $\bar{y}$, it is sufficient that the following equation

$$
s=\Lambda \rho+\int m\left(\nu_{r \rho, K}\right) d I(r)
$$

which is equation (2), has a unique root $\rho>0$. It is straightforward that the right-hand side of this equation is an increasing function of $\rho$ on $\mathbb{R}_{*}^{+}$, from 0 to $+\infty$. This guarantees the existence and the uniqueness of the solution.

Limiting invariant measure. The purpose then is to prove that this equilibrium point is the concentration point as $N$ tends to infinity of the invariant measure of process $Y^{N}$. A Lyapunov function $g$ of the dynamical system $(y(t))$ is a function which decreases along its trajectories, more precisely, for each $t \geq 0, \frac{d}{d t} g(y(t)) \leq 0$ with equality only if $y$ is the unique equilibrium point of $(y(t))$. Finding a Lyapunov function is a way to prove that the equilibrium point is the concentration point of the invariant measure of process $Y^{N}$ (see details for example in [12]). The following proposition provides a Lyapunov function. Let us recall some definition. For $\mu$ and $\mu^{\prime}$ probability measures on $\{0, \ldots, K\}$, $H\left(\mu / \mu^{\prime}\right)=\sum_{n=0}^{K} \mu_{n} \log \left(\mu_{n} / \mu_{n}^{\prime}\right)$ is the relative entropy of $\mu$ with respect to $\mu^{\prime}$.

Theorem 2 Function g defined on $\mathcal{Y}$ by $g(y)=h(y)-S(\rho(y))$, where $\rho(y)$ is defined by equation (6),

$$
\begin{gathered}
S(u)=u \Lambda-s \log u+\int_{] 0,1] \times \mathbb{N}} \log \frac{1-(u r)^{K+1}}{1-u r} d I(r, K), \\
h(y)=\int_{[0,1] \times \mathbb{N}} H\left(y(r, K, .) / \nu_{r \rho(y), K}\right) d I(r, K),
\end{gathered}
$$

and $\nu_{a, K}$ is defined in Theorem 1, is a Lyapunov function for the dynamical system $(y(t))$.

Scketch of the proof. The main argument is the decreasing of the relative entropy along the trajectories of a Markov process. Here, the dynamical process can be interpreted as a Markov process but with transitions depending on time. This adds a supplementary term, deriving the relative entropy, which is the second term of the expression of $g$.

Remark. Theorem 1 could be extended when $I$ has no more a finite support. The previous approach would work, with further technical arguments for proving the convergence to the dynamical system, and also that $g$ is a Lyapunov function. 


\subsection{The asymptotic of the partition function}

As the invariant measure has an explicit form, an analytical method can be considered. It consists in a saddle point method to obtain the asymptotic for $Z_{N}$ when $N$ gets large with $M / N$ which tends to $s$. If the capacity of the stations is infinite, the asymptotic was given in the paper by Kogan [8] , mentioned also in [9, Section 6] where a result similar to Theorem 1 appears.

Let $Z_{N}$ be indexed also by $M$. Substituting equation for $Z_{M, N}$,

$$
\sum_{M=0}^{+\infty} z^{M} Z_{M, N}=e^{z} \prod_{i=1}^{N} \frac{1-\left(z R_{i, N}\right)^{K_{i}+1}}{1-z R_{i, N}},|z|<1 .
$$

If $\Theta_{N}(u)=\sum_{M=0}^{+\infty}\left(u / R_{\max , N}\right)^{M} Z_{M, N}$ then

$$
\Theta_{N}(u)=e^{u / R_{\max , N}} \prod_{i=1}^{N} \frac{1-\left(u r_{i, N}\right)^{K_{i}+1}}{1-u r_{i, N}}
$$

which can be rewritten $\Theta_{N}(u) / u^{M}=e^{N S_{N}(u)}$, where

$$
S_{N}(u)=\frac{u}{N R_{\max , N}}-\frac{M}{N} \log u-\frac{1}{N} \sum_{i=1}^{N} \log \frac{1-\left(u r_{i, N}\right)^{K_{i}+1}}{1-u r_{i, N}} .
$$

By assumption $(W), S_{N}(u)$ converges to $S(u)$ given by equation (7). By derivation and some algebra,

$$
\begin{aligned}
S^{\prime}(u) & =\Lambda-\frac{s}{u}+\int_{] 0,1] \times \mathbb{N}}\left(\frac{r}{1-u r}-\frac{(K+1) r(u r)^{K}}{1-(u r)^{K+1}}\right) d I(r, K) \\
& =\Lambda-\frac{s}{u}+\frac{1}{u} \int_{] 0,1] \times \mathbb{N}} m\left(\nu_{u r, K}\right) d I(r, K) .
\end{aligned}
$$

Thus, $S^{\prime}(u)=0$ if and only if $\mathrm{u}$ is a root of equation (2). A saddle point method will give that, as $N \rightarrow+\infty$ and $M / N \rightarrow s$,

$$
1 / N \cdot \log \left(Z_{M, N} / R_{\max , N}\right) \sim S(\rho) .
$$

It is worth noting the presence of $S$ both in the partition function asymptotic and the Lyapunov function.

\section{Model II}

In this section, a second model is proposed. In model II, the total arrival process to the system is exactly the same as in Model I: a Poisson process with rate $\sum_{i=1}^{N} \lambda_{i, N}$, but users arrive only at non-empty stations, with a rate at a non empty station $i$ proportional to $\lambda_{i, N}$. Thus he always find a bike and takes it for an exponential time with parameter $\mu_{N}$, then returns it at a non saturated station $j$, with probability proportional to $p_{j, N}$. It takes into account the case where the users have information about the problematic stations and avoid them.

In model II, the Markovian dynamics are no more reversible. Moreover, the invariant distribution is out of reach and does not in general depend only on the utilization of the stations. But a result, similar to Theorem 1, is only partially obtained for model II. The idea is , to introduce in the empirical measure a deterministic coordinate more general than the utilization, to obtain the behavior of the system as it gets large by a mean field approach. What can be proved is given by the following proposition. 
Theorem 3 Assume that it exists I probability measure on $\mathbb{R}^{3} \times \mathbb{N}$ such that

$$
(W) \quad \frac{1}{N} \sum_{i=1}^{N} \delta_{\left(\lambda_{i, N}, N p_{i, N}, \mu_{N}, K_{i, N}\right)} \rightarrow I .
$$

If I has a finite support, as $N$ and $M$ tend to infinity with $M / N$ tending to some $s$, the empirical measure process converges to a dynamical process. There exists an equilibrium point $\bar{y}$ and for $x=(\lambda, \tilde{p}, \mu, K)$, $\bar{y}(x,$.$) has a geometric distribution with parameter r$, where $r=p \mu / \lambda$ and $\rho$ is a root of

$$
s=\int m\left(\nu_{r \rho, K}\right) d I(x)+\rho \frac{\int \lambda\left(1-\nu_{r \rho, K}(K)\right) d I(x)}{\int \lambda\left(1-\nu_{r \rho, K}(0)\right) d I(x)} .
$$

In the homogeneous case, the equilibrium point is unique and is the concentration point of the sequence of the invariant measures of the number of bikes at a station.

Remark. The uniqueness of the equilibrium point and the concentration of the invariant measures are conjectured for the cluster case (i.e. when $I$ has a finite support).

Proof:

Non reversible dynamics. The empirical measure process $\left(Y^{N}(t)\right)$ to consider is defined by

$$
Y^{N}(t)=\frac{1}{N} \sum_{i=1}^{N} \delta_{\left(x_{i, N}, X_{i, N}(t)\right)}
$$

where $x_{i, N}=\left(\lambda_{i, N}, N p_{i, N}, \mu_{N}, K_{i, N}\right)$ is called the parameter of station $i$. It is also a Markov process, on a finite state space $\mathcal{Y}^{N}$ included in $\mathcal{Y}=\mathcal{P}\left(\mathbb{R}^{3} \times \mathbb{N}\right)$ with transitions, if $x=(\lambda, \tilde{p}, \mu, K)$,

$$
\begin{array}{lr}
y \rightarrow y+\frac{1}{N}\left(\delta_{(x, n-1)}-\delta_{(x, n)}\right) & \frac{N \lambda y(x, n) 1_{n>0}}{\sum_{\left(x^{\prime}, n^{\prime}\right)} \lambda^{\prime} y\left(x^{\prime}, n^{\prime}\right) 1_{n^{\prime}>0}} \\
y \rightarrow y+\frac{1}{N}\left(\delta_{(x, n+1)}-\delta_{(x, n)}\right) & \frac{\tilde{p} y(x, n) 1_{n<K}}{\sum_{\left(x^{\prime}, n^{\prime}\right)} \tilde{p}^{\prime} y\left(x^{\prime}, n^{\prime}\right) 1_{n^{\prime}<K}} \mu\left(S-\sum_{\left(x^{\prime}, n^{\prime}\right)} n^{\prime} y\left(x^{\prime}, n^{\prime}\right)\right) .
\end{array}
$$

The first transition is an arrival at a station with parameter $x$ and with $n$ bikes, $n>0$. The second one is a return of a bike in a station with parameter $x$ and with $n$ bikes, $n<K$.

Under assumption $(W)$, for $I$ with finite support, standard arguments give that the empirical measure process converges to the unique solution of

$$
\begin{gathered}
\dot{y}=\int\left(\delta_{(x, n-1)}-\delta_{(x, n)}\right) \frac{\lambda 1_{n>0}}{\int_{\left(x^{\prime}, n^{\prime}\right)} \lambda^{\prime} 1_{n^{\prime}>0} d y\left(x^{\prime}, n^{\prime}\right)}+ \\
\left(\delta_{(x, n+1)}-\delta_{(x, n)}\right) \frac{\tilde{p} \mu 1_{n<K}}{\int_{\left(x^{\prime}, n^{\prime}\right)} \tilde{p}^{\prime} 1_{n^{\prime}<K} d y\left(x^{\prime}, n^{\prime}\right)}\left(s-m\left(y_{2}(t)\right)\right) d y(x, n) .
\end{gathered}
$$

Equilibrium point. Let $\bar{y}$ be an equilibrium point of the dynamical system given by equation (10). As in Section 3.1, it can be proved that, for $x=(\lambda, \tilde{p}, \mu, K), \bar{y}(x,$.$) has a geometric distribution with$ 
parameter $r \rho$, where $r=p \mu / \lambda$ and $\rho$ is a root of equation (9). Thus, using the closed-form expressions for $\nu_{a, K}(0)$ and $\nu_{a, K}(K), \rho$ satisfies:

$$
s=\int m\left(\nu_{r \rho, K}\right) d I(r)+\left(\int \lambda \frac{1-(r \rho)^{K}}{1-(r \rho)^{K+1}} I(d x)\right) /\left(\int \lambda r \frac{1-(r \rho)^{K}}{1-(r \rho)^{K+1}} I(d x)\right) .
$$

For the homogeneous case, it is then straightforward that equation (11) has a unique root $\rho$ because, in this case, its right-hand side has a first term which reduces to a constant, $1 / r$, thus is increasing from 0 to $K$ when $\rho$ goes from 0 to $+\infty$. It is conjectured in the cluster case.

Limiting invariant measure. For the homogeneous model, we refer to [5] for details. The key argument is that $m(\bar{y})=s-\lambda / \mu$ is constant, which is no more valid even in the cluster case.

\section{Performance}

Performance of the system is measured by the stationary probability that a station is problematic, i.e. has no bikes, which means the loss of arriving users, or saturated, i.e. with no lockers available, so users cannot return their bike. For model I, by Theorem 1, when the system gets large, if the station has relative utilization $r$ and capacity $K$, this probability tends to $\bar{y}(r, K, 0)+\bar{y}(r, K, K)$. Recall that $\bar{y}(r, K,)=.\nu_{\rho r, K}$ where $\rho$ is the root of equation (2). Model II is also detailed.

\subsection{Model I in the one cluster case}

Proposition 1 Consider model I in the one cluster case.

(i) The limiting proportion of problematic stations as a function of $s$ is given by the parametric curve

$$
\rho \mapsto\left(s(\rho), \nu_{\rho, K}(0)+\nu_{\rho, K}(K)\right)=\left(\frac{\lambda}{\mu} \rho+m\left(\nu_{\rho, K}\right), \frac{1-\rho}{1-\rho^{K+1}}\left(1+\rho^{K}\right)\right) .
$$

(ii) It is minimal when $s=K / 2+\lambda / \mu$ and the minimum is equal to $2 /(K+1)$.

(iii) As $K$ grows, the performance around $s=K / 2+\lambda / \mu$ becomes flatter and insensitive to $\lambda / \mu$.

Proof: Assertion $(i)$ is a simple consequence of Theorem 1. For assertion (ii), the intuitive argument is that the behavior of this geometric distribution $\nu_{\rho, K}$ for the number of bikes per station depends on the position of $\rho$ with respect to 1 : more concentrated on small values if $\rho$ is less than one, on large values, up to $K$, if $\rho$ is greater than one. Indeed, it can be proved that

$$
\varphi(\rho)=\nu_{\rho, K}(0)+\nu_{\rho, K}(K)
$$

has a minimum at 1 which is $2 /(K+1)$. And thus $s(1)=K / 2+\lambda / \mu$ is the optimal proportion of bikes per station. For (iii), it can be proved that, for $\psi=\varphi \circ s^{-1}$,

$$
\psi^{\prime \prime}(s(1))=\frac{K(K-1)}{3(K+1)\left(\lambda / \mu+K^{2} / 12+K / 6\right)^{2}} \geq \frac{48}{K(K+2)^{2}} .
$$

It gives that the performance curve can never be sharp at the optimal value, for the range of values considered here, i.e. $K \geq 20$. See [5] for details.

In practical situations, a system with 30 lockers per station would lead to a proportion of problematic stations of at least $2 / 31 \approx 6.5 \%$. 


\subsection{Model I in the cluster case}

When we have multiple clusters, the proportion of problematic stations in a cluster $c$ can be expressed as a function of $\rho$ and is equal to

$$
\nu_{\rho r_{c}, K_{c}}(0)+\nu_{\rho r_{c}, K_{c}}\left(K_{c}\right)=\frac{1-\rho r_{c}}{1-\left(\rho r_{c}\right)^{K_{c}+1}}\left(1+\left(\rho r_{c}\right)^{K_{c}}\right) .
$$

The study of the one cluster case gives that this quantity has a minimum $2 /\left(K_{c}+1\right)$ for $\rho r_{c}$ equal to 1 and, in this case, the proportion of problematic stations in cluster $c^{\prime}$ is exactly $\nu_{r_{c^{\prime}} / r_{c}, K_{c^{\prime}}}(0)+\nu_{r_{c^{\prime}} / r_{c}, K_{c^{\prime}}}\left(K_{c^{\prime}}\right)$.

As for the one cluster case, the proportion of problematic stations as a function of $s$ can be plotted using the parametric curve given by, for $\rho>0$,

$$
\rho \mapsto\left(\Lambda \rho+\sum_{c=1}^{C} \alpha_{c} m\left(\nu_{\rho r_{c}, K_{c}}\right), \sum_{c=1}^{C} \alpha_{c} \frac{1-\rho r_{c}}{1-\left(\rho r_{c}\right)^{K_{c}+1}}\left(1+\left(\rho r_{c}\right)^{K_{c}}\right)\right) .
$$

\subsection{Model II}

When studying the equilibrium point of the system, the only difference with model I studied in Sections 2 and 3 is that $\rho$ is given by equation (11), conjectured to have a unique solution. Therefore, we can plot easily the performance of the system by a parametric curve similar to (12) but where $s(\rho)$ in (12) is given by equation (11). For the one cluster case, it means simply to replace $\lambda / \mu \rho$ by $\lambda / \mu$ in $s(\rho)$ and, moreover, when $s \notin[\lambda / \mu, K+\lambda / \mu]$, the proportion of problematic stations is one.

It shows that the proportion of problematic stations has a similar shape for both models. Indeed, as before, the minimal proportion is $2 /(K+1)$ and is obtained for $s=K / 2+\lambda / \mu$. When $s$ is not equal to $K / 2+\lambda / \mu$, this proportion will be higher than in model $\mathrm{I}$.

This shows that although forcing people to go to a non-saturated or non-empty station reduces the unhappy users since everyone can take or leave a bike at anytime, it makes the system more congested and does not improve overall performance. A user that is not aware of the state of the system will have more problems in this system than in the first one.

\subsection{An example.}

This is illustrated for a two cluster case where the performance for both models is compared. The two clusters have the same size $N / 2$, the travel times have the same exponential distribution with parameter 1 , the probability to join each station $p_{1, N}=p_{2, N}=1 / N$ and the number of slots $K_{1}=K_{2}=30$ is the same but the arrival rates are $\lambda_{1}=1.3$, respectively 2 , while $\lambda_{2}=1$. Figure 1 is obtained. In Figure 2 , the proportion of empty and full stations is detailed. For example, it can be seen, from Figure 2, that even when the system is close to symmetry $\left(\lambda_{1} / \lambda_{2}=1.3\right)$, if the number of bikes is optimal, near 13 , a user going from an underloaded to an overloaded station has more than $25 \%$ chance of not finding a bike and more than $10 \%$ of finding a saturated station. This example shows that even slight asymmetry implies poor system performance.

\section{Conclusion and future work}

By extending the mean field methods for a model with clusters, we obtained performance of an inhomogeneous bike sharing system. Our argument would apply to other large networks. To make the model 

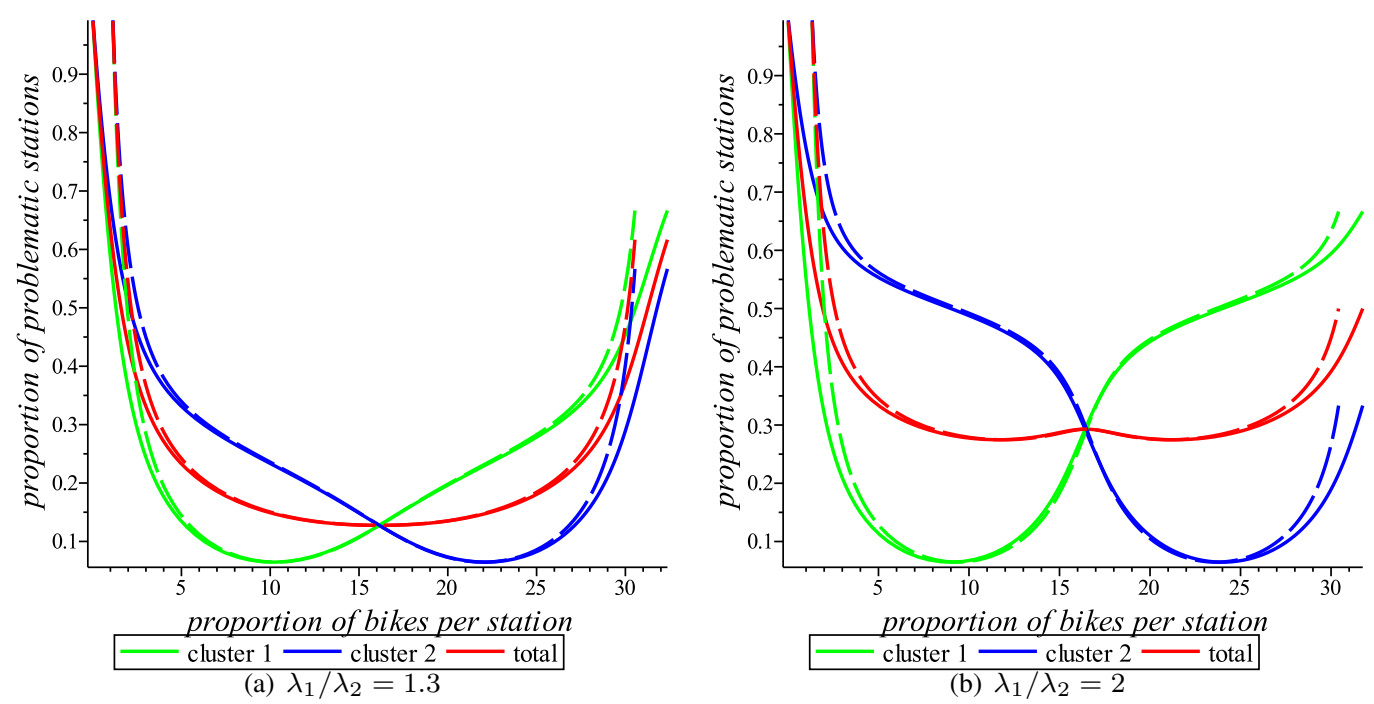

Fig. 1: Performance of the system as a function of the number of bikes in the city with two areas that have different arrival rates for model I (solid) and model II (dash).

analytically tractable, the way to return the bike then is simplified and we do not take into account geometry. For example, in a real system, a user finding a saturated station would search for an available spot in a local neighborhood. Our simulations on more realistic models, not presented here, suggest that the performance is very close. Future work would include evaluation of the influence of geometry.

Moreover, due to intrinsically poor performance, there is actually a pressing need to find efficient algorithms based on incentives to the users or relying on redistribution of bikes by trucks. This question has been investigated in [5] for an homogeneous system. Designing algorithms for inhomogeneous systems and studying then the system behavior is a challenging problem.

\section{References}

[1] Pierre Borgnat, Céline Robardet, Jean-Baptiste Rouquier, Patrice Abry, Eric Fleury, and Patrick Flandrin. Shared bicycles in a city: A signal processing and data analysis perspective. June 2011.

[2] R. W. R. Darling and J. R. Norris. Structure of large random hypergraphs. Ann. Appl. Probab., 15(1A):125-152, 2005.

[3] Paul Dupuis and Markus Fisher. On the construction of lyapunov functions for nonlinear markov processes via relative entropy. submitted for publication, 2011.

[4] Philippe Flajolet and Robert Sedgewick. Analytic Combinatorics. Cambridge University Press, 2009.

[5] Christine Fricker and Nicolas Gast. Incentives and regulations in bike-sharing systems with stations of finite capacity. arXiv:1201.1178v1, January 2012. 

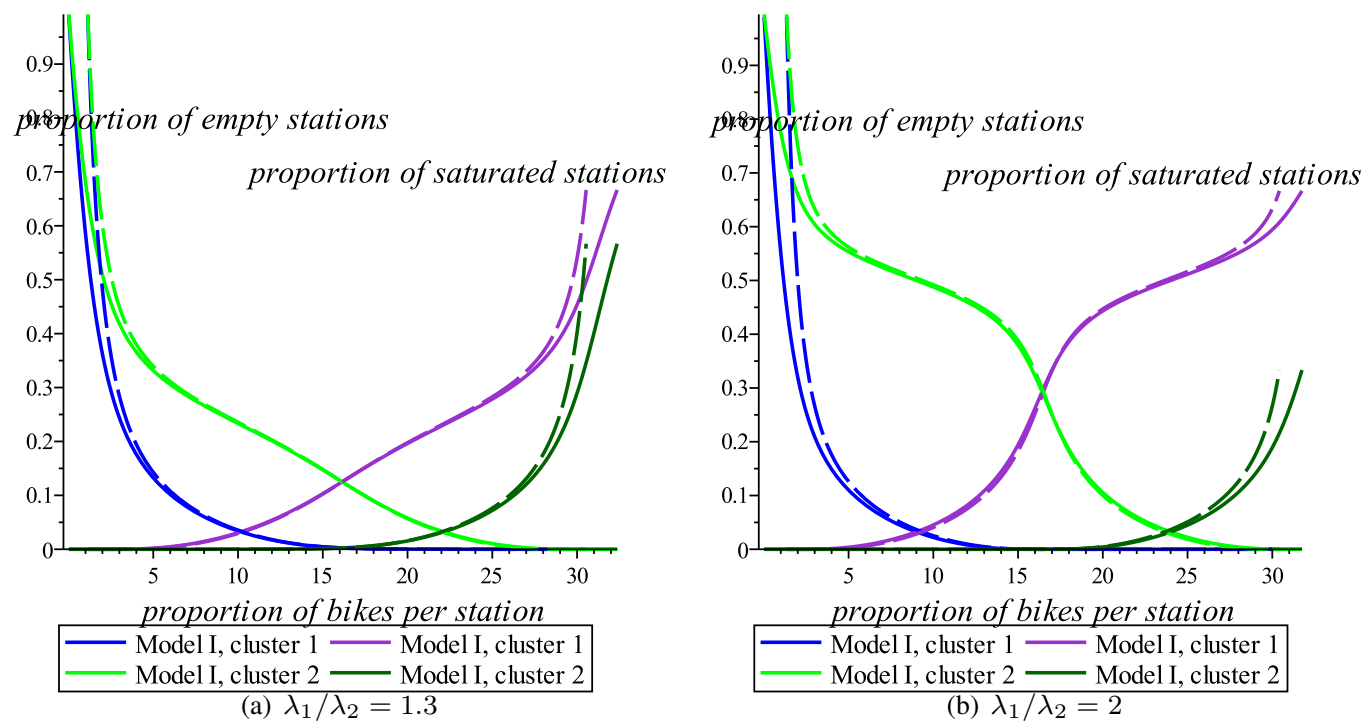

Fig. 2: Proportion of empty and full stations as a function of the number of bikes in a city with two areas that have different arrival rates for model I (solid) and model II (dash).

[6] Nicolas Gast and Bruno Gaujal. A mean field model of work stealing in large-scale systems. ACM SIGMETRICS Performance Evaluation Review, 38(1):13-24, 2010.

[7] David K. George and Cathy H. Xia. Asymptotic analysis of closed queueing networks and its implications to achievable service levels. SIGMETRICS Performance Evaluation Review, 38(2):3$5,2010$.

[8] Yaakov Kogan. Another approach to asymptotic expansions for large closed queueing networks. Oper. Res. Lett., 11(5):317-321, 1992.

[9] Vadim A. Malyshev and Andrei V. Yakovlev. Condensation in large closed Jackson networks. Ann. Appl. Probab., 6(1):92-115, 1996.

[10] Michael Mitzenmacher. The Power of Two Choices in Randomized Load Balancing. PhD thesis, Berkeley, 1996.

[11] Michael Mitzenmacher. Studying balanced allocations with differential equations. Combin. Probab. Comput., 8(5):473-482, 1999.

[12] Danielle Tibi. Metastability in communications networks. arXiv:1002.07/96v1. 